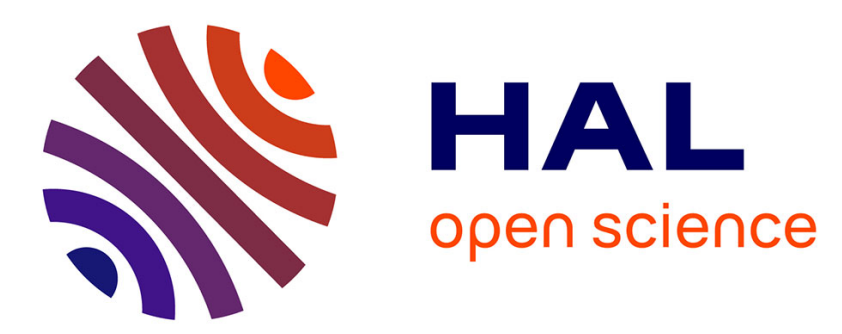

\title{
From natural to mixed convection in horizontal and differentially heated annular ducts: linear stability analysis
}

Eric Chénier, G. Petrone, Guy Lauriat

\section{- To cite this version:}

Eric Chénier, G. Petrone, Guy Lauriat. From natural to mixed convection in horizontal and differentially heated annular ducts: linear stability analysis. International Journal of Heat and Mass Transfer, 2011, 54 (23-24), pp.5100-5108. 10.1016/j.ijheatmasstransfer.2011.07.028 . hal-00682950

\section{HAL Id: hal-00682950 \\ https://hal.science/hal-00682950}

Submitted on 27 Mar 2012

HAL is a multi-disciplinary open access archive for the deposit and dissemination of scientific research documents, whether they are published or not. The documents may come from teaching and research institutions in France or abroad, or from public or private research centers.
L'archive ouverte pluridisciplinaire HAL, est destinée au dépôt et à la diffusion de documents scientifiques de niveau recherche, publiés ou non, émanant des établissements d'enseignement et de recherche français ou étrangers, des laboratoires publics ou privés. 


\title{
From natural to mixed convection in horizontal and differentially heated annular ducts: linear stability analysis
}

\author{
E. Chénier ${ }^{\mathrm{a}, *}$, G. Petrone ${ }^{\mathrm{b}}$, G. Lauriat ${ }^{\mathrm{a}}$ \\ ${ }^{a}$ Laboratoire Modélisation et Simulation Multi Echelle, UMR 8208 CNRS, Université Paris-Est \\ Marne-la-Vallée, Bâtiment Lavoisier, 5 Boulevard Descartes, 77454 MARNE-LA-VALLEE Cedex 2, \\ FRANCE \\ ${ }^{b}$ Dipartimento di Ingegneria Industriale e Meccanica, Università degli Studi di Catania, V.le A. Doria 6, \\ 95125 Catania, ITALIA
}

\begin{abstract}
Linear stability analysis of a fully developed mixed convection flow of air in an annular horizontal duct is numerically investigated for the radius ratio $R=1.2$, a Péclet and a Rayleigh number less than 200 and 6000, respectively. An iterative method is developed to enable the convergence of the dimensionless parameters to their marginal values at the transition. New mixed convection flows are highlighted that are highly correlated with those obtained in natural convection problems under the assumption of two dimensionality. The synthesis of our results on the transitions permits us to build the map of stability for the steady and established mixed convection flows and clearly shows the occurrence of multiplicity of solutions for some couples of Rayleigh and Péclet numbers.
\end{abstract}

Keywords: mixed convection, horizontal annulus, stability analysis

\section{Nomenclature}

$\begin{array}{ll}\boldsymbol{e}_{3} & \text { vertical unit vector pointing upwards } \\ f & =\eta r+1 \\ g & \text { gravity acceleration, } m / s^{2} \\ \mathrm{i} & \text { pure imaginary number } \\ k & \text { real wavenumber } \\ p & \text { pressure } \\ \mathrm{Pe} & \text { Péclet number, }=\frac{\bar{w}^{*}\left(r_{o}^{\star}-r_{i}^{\star}\right)}{\alpha} \\ \operatorname{Pr} & \text { Prandtl number, }=\frac{\nu}{\alpha} \\ r & \text { reduced radial coordinate, }=\frac{r^{\star}-r_{i}^{\star}}{r_{o}^{\star}-r_{i}^{\star}} \\ (r, \theta, z) & \text { cylindrical coordinates }\end{array}$

${ }^{*}$ Corresponding author

Email addresses: Eric.Chenier@univ-paris-est.fr (E. Chénier), gpetrone@diim.unict.it (G. Petrone), Guy.Lauriat@univ-paris-est.fr (G. Lauriat)

Submitted to International Journal of Heat and Mass Transfer(2011) 
$R$

Ra

$\widehat{\operatorname{Ra}}(k)$

$r_{i}^{\star}$

$r_{o}^{\star}$

T

$\boldsymbol{v}$

$\bar{w}$

\section{Subscripts}

0

$c$

$k$

$k, \lambda$

\section{Superscripts}

1 st

2nd

$*$

Greek symbols

$\alpha$

$\beta$

$\delta \boldsymbol{v}$

$\Delta P$

$\eta$

$\lambda$

$\lambda_{r}$

$\lambda_{r}^{M}$

$\lambda_{i}$

$\lambda_{i}^{M}$

$\nu$

$\rho$ radius ratio, $=r_{o}^{\star} / r_{i}^{\star}$

Rayleigh number, $=\frac{g \beta\left(T^{*}\left(r_{i}^{\star}\right)-T^{*}\left(r_{o}^{\star}\right)\right)\left(r_{o}^{\star}-r_{i}^{\star}\right)^{3}}{\nu \alpha}$

critical Rayleigh number function of the $k$

inner radius, $m$

outer radius, $m$

temperature

velocity vector, $=u \boldsymbol{e}_{r}+v \boldsymbol{e}_{\theta}+w \boldsymbol{e}_{z}$

mean axial velocity

steady basic solution

threshold value

component in the Fourier space

component in the Fourier and Laplace spaces

first branch of solutions

second branch of solutions

dimensional variable

thermal diffusivity, $\mathrm{m}^{2} / \mathrm{s}$

expansion coefficient, $K^{-1}$

velocity vector for the perturbation, $=\delta u \boldsymbol{e}_{r}+\delta v \boldsymbol{e}_{\theta}+\delta w \boldsymbol{e}_{z}$

constant axial pressure gradient

relative annular gap, $=R-1$

complex eigenvalue, $=\lambda_{r}+i \lambda_{i}$

growth rate

maximum growth rate

pulsation

pulsation of the complex eigenvalue having $\lambda_{r}^{M}$ as real part

kinematic viscosity, $\mathrm{m}^{2} / \mathrm{s}$

density, $\mathrm{kg} / \mathrm{m}^{3}$

\section{Introduction}

Natural, forced and mixed convection in horizontal annuli is a fundamental issue of interest and has been extensively studied. This interest stems from the wide range of related engineering applications such as thermal energy storage systems, heat exchangers, transmission cables, solar collectors, etc.

Natural convection in differentially heated horizontal annuli inspired numerous studies because of the role of curvature on the birth of thermal instabilities. Although early ex- 
perimental work dates from 1931 (Beckmann [1]), it took forty years to have a qualitative description of flows depending on the Grashof number and radius ratio (Grigull and Hauf [2], Powe et al. [3]). With the increase in computational resources, numerous numerical simulations were carried out, but mainly under the assumption of two-dimensional flows, invariant in the axial direction. These studies show that two-dimensional flow, which develops in the form of two large symmetrical and crescent-shaped cells, undergoes a Rayleigh-Bénard instability with the increase in the Rayleigh number, for radius ratio in the range $1.2 \leq R \leq 2$ (see Petrone et al. [4] for example). The supercritical flow pattern is then made of one or two pairs of additional convection rolls located at the top of the annulus, thereby enhancing heat transfer rate between the cylinder walls. However, these two-dimensional flows turn out to be unstable with respect to three-dimensional perturbations [5-8]. A critical review of buoyancy-induced flow transitions in horizontal annuli can be found in a recent paper by Angeli et al. [9].

Forced convection, and to a lesser extent mixed convection, have been the subject of many analytical, experimental and numerical investigations, concerning both the entrance regions (dynamical and thermal) and the heat transfer for fully developed flows [10]. Graetz [11] (1883), Nusselt [12] (1910) and later on Lévêque [13] (1928) were interested in the issue of the developing thermal regime for a fluid flowing in the laminar established regime in a pipe whose walls were maintained at uniform temperature. In this model, the axial diffusion is neglected, such an assumption is justified when the Péclet number is sufficiently high (Pe > 100). Based on similar assumptions, the works of Lundberg et al. [14] and Shah and London [15] provided a comprehensive study on the establishment of thermal regime in an annular duct for several combinations of flow conditions and temperature applied at pipe walls. With similar assumptions, Kakaç and Yücel [16] studied the laminar flow heat transfer in annuli with simultaneous development of velocity and temperature fields. For low values of Péclet number, both axial diffusion $[17,18]$ and free convection $[19,20]$ become not negligible in respect of the establishment length value, which is also strongly affected by thermal conditions applied at the walls. Amongst the papers dealing with the entrance regions, a few are devoted to experimental investigations (see for example the recent paper of Mohammed et al. [21] and references herein). Finally, to our best knowledge, few numerical studies were focused on the influence of natural convection in dynamically and thermally fully developed flows in annular ducts for very low Reynolds number values, and only for large radii ratios [22].

Despite these numerous studies, there are still many aspects that need to be explored or thorough, especially concerning the effects of an axial flow on the multicellular secondary flows induced by the buoyancy force in narrow annular spaces. To this aim, a linear stability analysis of the fully developed flow is performed for air flowing in an annular pipe of fixed radius ratio $R=1.2$. The rest of the paper is structured as follows. Section 2 is devoted to the presentation of the governing equations for the basic flow and the perturbed states. A numerical method, suitable for calculating the transition thresholds in a plane of Rayleigh number and Péclet number, is presented. It is built around an iterative method, coupling the calculation of the basic steady flow and determination of the dominant spectrum of the linearized problem. The iterative process, involving the wavenumber and Rayleigh number, 
is based on approximate Newton methods for which the derivatives are substituted by simple algebraic relations. Section 3 emphasizes the close link between our previous works about pure free convection and the nature of the flow that develops in the cross sections of fluid flow in mixed convection. The sensitivity of the critical Rayleigh number is studied as a function of the Péclet number, and it is shown that topologies which are linearly unstable in natural convection turn out to be stable in mixed convection. In particular we show that multiple solutions are simultaneously stable for certain ranges of the couple(Pe, Ra). Finally, a conclusion is drawn that highlights the main issues of this work.

\section{Equations}

\subsection{Physical model}

The horizontal annular pipe is confined by two co-axial and infinite cylinders of radii $r_{i}^{\star}$ and $r_{o}^{\star}>r_{i}^{\star}$ (Fig.1). The temperature of the inner and outer cylinders is kept constant

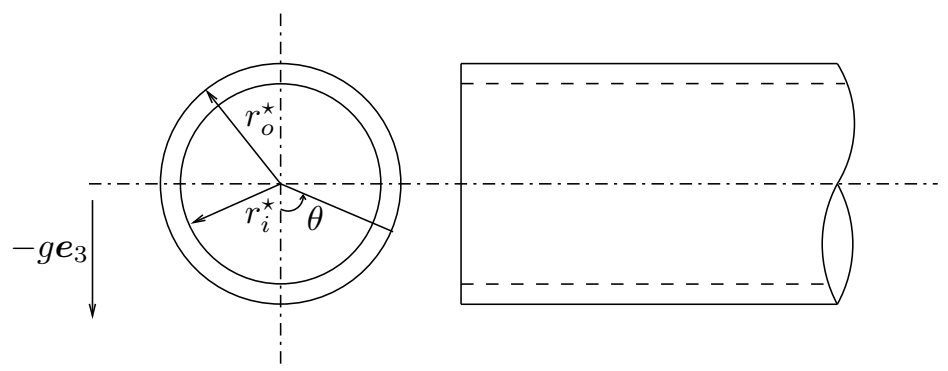

Figure 1: Geometry

such that $T^{\star}\left(r_{o}^{\star}\right)<T^{\star}\left(r_{i}^{\star}\right)$. The fluid flow is assumed incompressible with constant physical properties except the density in the buoyancy term. The axial coordinate is scaled by the annulus gap $r_{o}^{\star}-r_{i}^{\star}$, the velocity components by the mean axial velocity $\bar{w}^{*}$, the dynamical pressure by $\rho\left(\bar{w}^{*}\right)^{2}$ and the time by $\left(r_{o}^{\star}-r_{i}^{\star}\right) / \bar{w}^{*}$. We also introduce the dimensionless temperature difference $T=\left(T^{\star}-T_{r}^{\star}\right) /\left(T^{\star}\left(r_{i}^{\star}\right)-T^{\star}\left(r_{o}^{\star}\right)\right)$ with $T_{r}^{\star}=\left(T^{\star}\left(r_{i}^{\star}\right)+T^{\star}\left(r_{o}^{\star}\right)\right) / 2$, and the reduced radial coordinate $r=\left(r^{\star}-r_{i}^{\star}\right) /\left(r_{o}^{\star}-r_{i}^{\star}\right)$.

To shorten the writing of equations presented in this article, and to emphasize the role of two-dimensional flows that develop in planes transverse to the axis of the cylinders, the partial derivative operators have been split into an implicit part coupling the radial and azimuthal directions, and symbolically represented by " $\nabla_{2 d} \cdot$ ", " $\bar{\nabla}_{2 d} \cdot "$, " $\nabla_{2 d}^{2} ", " \overline{\nabla_{2 d}^{2}}$ " and " $\nabla_{2 d}$ ", and an explicit part that deals only with the axial derivatives. Thus, by combining the radial and azimuthal components of the momentum equation into a single vectorial relation (see Eq.(1b)), we obtain the three-dimensional Navier-Stokes and energy equations as follows:

$$
\boldsymbol{\nabla}_{2 d} \cdot \boldsymbol{v}+\frac{\partial(f w)}{\partial z}=0
$$




$$
\begin{aligned}
& \frac{\partial}{\partial t}(f u) \boldsymbol{e}_{r}+\frac{\partial}{\partial t}(f v) \boldsymbol{e}_{\theta}+\overline{\nabla_{2 d}} \cdot(\boldsymbol{v} \otimes \boldsymbol{v})+ \frac{\partial(f w u)}{\partial z} \boldsymbol{e}_{r}+\frac{\partial(f w v)}{\partial z} \boldsymbol{e}_{\theta}=-\boldsymbol{\nabla}_{2 d} p+\frac{\operatorname{RaPr}}{\mathrm{Pe}^{2}} f T \boldsymbol{e}_{3} \\
&+\frac{\operatorname{Pr}}{\operatorname{Pe}}\left(\overline{\nabla_{2 d}^{2}} \boldsymbol{v}+\frac{\partial}{\partial z}\left(f \frac{\partial u}{\partial z}\right) \boldsymbol{e}_{r}+\frac{\partial}{\partial z}\left(f \frac{\partial v}{\partial z}\right) \boldsymbol{e}_{\theta}\right) \\
& \frac{\partial}{\partial t}(f w)+\nabla_{2 d} \cdot(w \boldsymbol{v})+\frac{\partial\left(f w^{2}\right)}{\partial z}=-f \frac{\partial p}{\partial z}+\frac{\operatorname{Pr}}{\operatorname{Pe}}\left(\nabla_{2 d}^{2}+\frac{\partial}{\partial z}\left(f \frac{\partial}{\partial z}\right)\right) w \\
& \frac{\partial}{\partial t}(f T)+\nabla_{2 d} \cdot(T \boldsymbol{v})+\frac{\partial(f T w)}{\partial z}=\frac{1}{\operatorname{Pe}}\left(\nabla_{2 d}^{2}+\frac{\partial}{\partial z}\left(f \frac{\partial}{\partial z}\right)\right) T
\end{aligned}
$$

with the following definitions for the two-dimensional operators:

- divergence of the vector field $X \boldsymbol{v}$

$$
\nabla_{2 d} \cdot(X \boldsymbol{v})=\frac{\partial(f X u)}{\partial r}+\frac{\partial(\eta X v)}{\partial \theta}
$$

- divergence of the tensorial field $\boldsymbol{v} \otimes \boldsymbol{v}$

$$
\overline{\nabla_{2 d}} \cdot(\boldsymbol{v} \otimes \boldsymbol{v})=\left(\boldsymbol{\nabla}_{2 d} \cdot(u \boldsymbol{v})-\eta v^{2}\right) \boldsymbol{e}_{r}+\left(\boldsymbol{\nabla}_{2 d} \cdot(v \boldsymbol{v})+\eta u v\right) \boldsymbol{e}_{\theta}
$$

- Laplacian of the scalar variable $X$

$$
\nabla_{2 d}^{2} X=\frac{\partial}{\partial r}\left(f \frac{\partial X}{\partial r}\right)+\frac{\partial}{\partial \theta}\left(\frac{\eta^{2}}{f} \frac{\partial X}{\partial \theta}\right)
$$

- Laplacian of the vector field $\boldsymbol{v}$

$$
\overline{\nabla_{2 d}^{2}} \boldsymbol{v}=\left(\nabla_{2 d}^{2} u-\frac{2 \eta^{2}}{f} \frac{\partial v}{\partial \theta}-\frac{\eta^{2} u}{f}\right) \boldsymbol{e}_{r}+\left(\nabla_{2 d}^{2} v+\frac{2 \eta^{2}}{f} \frac{\partial u}{\partial \theta}-\frac{\eta^{2} v}{f}\right) \boldsymbol{e}_{\theta}
$$

- gradient of the pressure $p$

$$
\nabla_{2 d} p=f \frac{\partial p}{\partial r} \boldsymbol{e}_{r}+\eta \frac{\partial p}{\partial \theta} \boldsymbol{e}_{\theta}
$$

where $\eta=R-1$, with $R=r_{o}^{\star} / r_{i}^{\star}$, and $f=\eta r+1$ are geometric parameters while $\boldsymbol{v}=$ $u \boldsymbol{e}_{r}+v \boldsymbol{e}_{\theta}+w \boldsymbol{e}_{z}$ and $p$ represent the dimensionless velocity and pressure. The dimensionless parameters $\mathrm{Ra}=g \beta\left(T^{\star}\left(r_{i}^{\star}\right)-T^{\star}\left(r_{o}^{\star}\right)\right)\left(r_{o}^{\star}-r_{i}^{\star}\right)^{3} /(\nu \alpha), \operatorname{Pr}=\nu / \alpha$ and $\mathrm{Pe}=\bar{w}^{*}\left(r_{o}^{\star}-r_{i}^{\star}\right) / \alpha$ stand for the Rayleigh, Prandtl and Péclet numbers, with $g, \beta, \nu$ and $\alpha$ the gravitational acceleration, the thermal expansion coefficient, the kinematic viscosity and the thermal diffusivity, respectively. The set of equations (1) is solved with the dimensionless boundary conditions at the solid walls:

$$
\begin{cases}\boldsymbol{v}=\mathbf{0}, T=+0.5 & \text { at } r=0 \\ \boldsymbol{v}=\mathbf{0}, T=-0.5 & \text { at } r=1\end{cases}
$$

with inflow and outflow boundary conditions and some prescribed initial conditions. 


\subsubsection{Fully developed flow - Basic solution}

The linear stability analysis is performed for a steady and fully developed fluid flow: under this assumption the time and axial derivatives for velocity and temperature cancel in Eqs (1). Because the flow is considered totally established, the pressure gradient in any transversal section of the pipe does not depend on the axial coordinate, and furthermore the axial pressure gradient along the pipe is constant. Thus, the dimensionless pressure term is rewritten as $p(r, \theta, z)=\Delta P \times z+p_{0}(r, \theta)$ where $\Delta P$ is the constant dimensionless axial pressure gradient and $p_{0}(r, \theta)$ represents the fluctuation pressure in any transversal section of the duct. By indexing the basic flow by "0", equations (1a)-(1d) are reduced to the $(r, \theta)$-equations:

$$
\begin{gathered}
\nabla_{2 d} \cdot \boldsymbol{v}_{0}=0 \\
\overline{\nabla_{2 d}} \cdot\left(\boldsymbol{v}_{0} \otimes \boldsymbol{v}_{0}\right)=-\nabla_{2 d} p_{0}+\frac{\operatorname{RaPr}}{\mathrm{Pe}^{2}} f T_{0} \boldsymbol{e}_{3}+\frac{\operatorname{Pr}}{\mathrm{Pe}} \overline{\nabla_{2 d}^{2}} \boldsymbol{v}_{0} \\
\boldsymbol{\nabla}_{2 d} \cdot\left(w_{0} \boldsymbol{v}_{0}\right)=-f \Delta P+\frac{\mathrm{Pr}}{\mathrm{Pe}} \nabla_{2 d}^{2} w_{0} \\
\nabla_{2 d} \cdot\left(T_{0} \boldsymbol{v}_{0}\right)=\frac{1}{\mathrm{Pe}} \nabla_{2 d}^{2} T_{0}
\end{gathered}
$$

with $\boldsymbol{v}_{0}=u_{0}(r, \theta) \boldsymbol{e}_{r}+v_{0}(r, \theta) \boldsymbol{e}_{\theta}+w_{0}(r, \theta) \boldsymbol{e}_{z}, T_{0}(r, \theta)$ and $p_{0}(r, \theta)$, and partial differential operators defined by (2a)-(2e). The boundary conditions are identical to relations (3); inflow and outflow boundary conditions and initial conditions are disregarded. At this stage, the axial pressure gradient $\Delta P$, which is a priori unknown, must be set so that the dimensionless axial velocity $\bar{w}$ equals to one, as prescribed by our velocity scaling. Notice that, with the decrease in Ra, one can expect that $\Delta P$ becomes closer to the analytical value $-8 \eta^{2} \mathrm{Pr} / \mathrm{Pe} /\left(R^{2}+1-\left(R^{2}-1\right) / \ln R\right)$ obtained for fully developed forced convection flows (see Rohsenow et al. [10]). The next paragraph is devoted to the determination of $\Delta P$ for mixed flow configurations.

The calculation of the axial pressure gradient $\Delta P$ is based on two fundamental remarks.

1. The mass conservation (4a) as well as the momentum (4b) and the energy (4d) equations do not depend on either the axial velocity $w_{0}$ or the axial pressure gradient. Therefore, any change in $\Delta P$ or $w_{0}$ will not affect the temperature field, the radial and axial components of the velocity or the fluctuation pressure $p_{0}$.

2. In Eq. (4c), there exists a linear relation between $\Delta P$ and $w_{0}$, that is between $\Delta P$ and $\bar{w}$.

Thus, from a single computation performed choosing an arbitrary axial pressure gradient $\Delta P_{1} \neq 0$ and giving rise to a mean flow velocity $\bar{w}_{1}$, the correct axial pressure gradient providing the mean velocity value equal to 1 , as requested by the adopted procedure for scaling the velocity components, is simply $\Delta P=\Delta P_{1} / \bar{w}_{1}$. The axial velocity component needs also to be rescaled by $\bar{w}_{1}$, and no additional computation is required. 


\subsubsection{Perturbed state equations}

The flow consisting in the sum of the two-dimensional basic solution $\left(\boldsymbol{v}_{0}, p_{0}, T_{0}\right)$ and a small three-dimensional perturbation $\delta \boldsymbol{v}=\delta u \boldsymbol{e}_{r}+\delta v \boldsymbol{e}_{\theta}+\delta w \boldsymbol{e}_{z}, \delta p$ and $\delta T$ is also solution of equations (1a)-(1d). Taking into account the order of magnitude of disturbances, the nonlinear contributions are linearized with respect to perturbations. The Fourier transform in the axial direction $\left(\mathcal{F}: Y(z) \rightarrow Y_{k}=\mathcal{F}_{k}(Y)\right)$ is then applied to the linear equations where $k$ stands for a real wavenumber. Let us now define the transforms of the velocity, temperature and pressure perturbations in complex form as: $\delta \boldsymbol{v}_{k}(r, \theta, t)=\mathcal{F}(\delta \boldsymbol{v}(r, \theta, z, t)), \delta T_{k}(r, \theta, t)=$ $\mathcal{F}(\delta T(r, \theta, z, t))$ and $\delta p_{k}(r, \theta, t)=\mathcal{F}(\delta p(r, \theta, z, t))$. The resulting complex equations for the perturbations are written as follows:

$$
\begin{gathered}
0=\boldsymbol{\nabla}_{2 d} \cdot \delta \boldsymbol{v}_{k}+\mathrm{i} k f \delta w_{k} \\
\frac{\partial}{\partial t}\left(f \delta u_{k}\right) \boldsymbol{e}_{r}+\frac{\partial}{\partial t}\left(f \delta v_{k}\right) \boldsymbol{e}_{\theta}=\mathbf{R H S}^{(1)}\left(\delta \boldsymbol{v}_{k}, \delta p_{k}, \delta T_{k}\right) \\
\frac{\partial}{\partial t}\left(f \delta w_{k}\right)=\operatorname{RHS}^{(2)}\left(\delta \boldsymbol{v}_{k}, \delta p_{k}\right) \\
\frac{\partial}{\partial t}\left(f \delta T_{k}\right)=\operatorname{RHS}^{(3)}\left(\delta \boldsymbol{v}_{k}, \delta T_{k}\right)
\end{gathered}
$$

The right-hand sides of Eqs. (5b)-(5d) are defined as follows:

$$
\begin{array}{r}
\mathbf{R H S}^{(1)}\left(\delta \boldsymbol{v}_{k}, \delta p_{k}, \delta T_{k}\right)=-\overline{\boldsymbol{\nabla}_{2 d}} \cdot\left(\boldsymbol{v}_{0} \otimes \delta \boldsymbol{v}_{k}\right)-\overline{\boldsymbol{\nabla}_{2 d}} \cdot\left(\delta \boldsymbol{v}_{k} \otimes \boldsymbol{v}_{0}\right) \\
-\mathrm{i} k f\left(w_{0} \delta u_{k}+\delta w_{k} u_{0}\right) \boldsymbol{e}_{r}-\mathrm{i} k f\left(w_{0} \delta v_{k}+\delta w_{k} v_{0}\right) \boldsymbol{e}_{\theta} \\
-\boldsymbol{\nabla}_{2 d} \delta p_{k}+\frac{\operatorname{RaPr}}{\mathrm{Pe}^{2}} f \delta T_{k} \boldsymbol{e}_{3}+\frac{\operatorname{Pr}}{\mathrm{Pe}}\left(\overline{\nabla_{2 d}^{2}} \delta \boldsymbol{v}_{k}-k^{2} f \delta u_{k} \boldsymbol{e}_{r}-k^{2} f \delta v_{k} \boldsymbol{e}_{\theta}\right) \\
\mathrm{RHS}^{(2)}\left(\delta \boldsymbol{v}_{k}, \delta p_{k}\right)=-\boldsymbol{\nabla}_{2 d} \cdot\left(w_{0} \delta \boldsymbol{v}_{k}\right)-\boldsymbol{\nabla}_{2 d} \cdot\left(\delta w_{k} \boldsymbol{v}_{0}\right)-2 \mathrm{i} k f \delta w_{k} w_{0}-\mathrm{i} k f \delta p_{k} \\
+\frac{\operatorname{Pr}}{\operatorname{Pe}}\left(\nabla_{2 d}^{2}-k^{2} f\right) \delta w_{k} \\
\operatorname{RHS}^{(3)}\left(\delta \boldsymbol{v}_{k}, \delta T_{k}\right)=-\nabla_{2 d} \cdot\left(\delta T_{k} \boldsymbol{v}_{0}\right)-\boldsymbol{\nabla}_{2 d} \cdot\left(T_{0} \delta \boldsymbol{v}_{k}\right)-\mathrm{i} k f\left(\delta T_{k} w_{0}+T_{0} \delta w_{k}\right) \\
+\frac{1}{\mathrm{Pe}}\left(\nabla_{2 d}^{2}-k^{2} f\right) \delta T_{k}
\end{array}
$$

The boundary conditions at the solid walls and the initial values write

$$
\delta \boldsymbol{v}_{k}=\mathbf{0}, \delta T_{k}=0 \quad \text { at } r=0 \text { and } r=1
$$


and

$$
\delta \boldsymbol{v}_{k}=\delta \boldsymbol{v}_{k}\left(t=0^{+}\right), \delta T_{k}=\delta T_{k}\left(t=0^{+}\right)
$$

The evolution problem (5) associated with boundary and initial conditions, relations (7a) and $(7 \mathrm{~b})$ respectively, can also be expressed by using a Laplace transform for time $(\mathcal{L}$ : $\left.X(t) \rightarrow X_{\lambda}=\mathcal{L}_{\lambda}(X)\right)$ with $\lambda=\lambda_{r}+\mathrm{i} \lambda_{i}, \lambda_{r}, \lambda_{i}$ being real values and $\mathrm{i}^{2}=-1$. In that case the variables $\delta \boldsymbol{v}_{k}, \delta T_{k}$ and $\delta p_{k}$ located in the right-hand sides of equations (5a)-(5d) write $\delta \boldsymbol{v}_{k, \lambda}=\mathcal{L}_{\lambda}\left(\delta \boldsymbol{v}_{k}\right), \delta T_{k, \lambda}=\mathcal{L}_{\lambda}\left(\delta T_{k}\right)$ and $\delta p_{k, \lambda}=\mathcal{L}_{\lambda}\left(\delta p_{k}\right)$ and the temporal derivatives defined in the left-hand sides of equations (5b)-(5d) give $\left(\lambda \delta \boldsymbol{v}_{k, \lambda}-\delta \boldsymbol{v}_{k}\left(t=0^{+}\right)\right) f$ and $\left(\lambda \delta T_{k, \lambda}-\delta T_{k}\left(t=0^{+}\right)\right) f$. Therefore, the growth or reduction of the perturbation magnitude is provided by the algebraic sign of the largest real eigenvalue of the eigenproblem obtained by setting the initial disturbances $\delta \boldsymbol{v}_{k}\left(t=0^{+}\right)$and $\delta T_{k}\left(t=0^{+}\right)$to zero (namely the general solution of the problem):

$$
\begin{gathered}
0=\boldsymbol{\nabla}_{2 d} \cdot \delta \boldsymbol{v}_{k, \lambda}+\mathrm{i} k f \delta w_{k, \lambda} \\
\lambda\left(f \delta u_{k, \lambda} \boldsymbol{e}_{r}+f \delta v_{k, \lambda} \boldsymbol{e}_{\theta}\right)=\mathbf{R H S}^{(1)}\left(\delta \boldsymbol{v}_{k, \lambda}, \delta p_{k, \lambda}, \delta T_{k, \lambda}\right) \\
\lambda f \delta w_{k, \lambda}=\operatorname{RHS}^{(2)}\left(\delta \boldsymbol{v}_{k, \lambda}, \delta p_{k, \lambda}\right) \\
\lambda f \delta T_{k, \lambda}=\operatorname{RHS}^{(3)}\left(\delta \boldsymbol{v}_{k, \lambda}, \delta T_{k, \lambda}\right)
\end{gathered}
$$

provided with homogeneous boundary conditions on solid walls and with relations (6a)-(6c) for the right-hand side terms. Note that the eigenvalue problem (8) is generally determined by assuming a particular form for the perturbations of velocity $\left(\delta \boldsymbol{v}_{k, \lambda}(r, \theta) \exp (\mathrm{i} k z+\lambda t)\right)$, temperature $\left(\delta T_{k, \lambda}(r, \theta) \exp (\mathrm{i} k z+\lambda t)\right)$ and pressure $\left(\delta p_{k, \lambda}(r, \theta) \exp (\mathrm{i} k z+\lambda t)\right)$ in the equations linearized around the basic flow. This choice results therefore only from a resolution of the perturbed equations in Fourier and Laplace spaces, as shown above.

\subsection{Numerical methods}

\subsubsection{Discretization scheme}

Continuous equations are discretized on a structured and staggered grid by a finite volume method. The spatial derivatives are approximated by a second-order centered scheme. The control volume $\left[r_{i} ; r_{i+1}\right] \times\left[\theta_{j} ; \theta_{j+1}\right]$ with $(i, j) \in\left(\mathbb{N}^{+}\right)^{2}$ for the pressure and the temperature is defined by

$$
\begin{aligned}
& r_{i}=\frac{i-1}{N_{r}}, 1 \leq i \leq N_{r}+1 \\
& \theta_{j}= \begin{cases}\frac{\exp \left(2 \pi c_{\theta} \frac{j-1}{N_{\theta}}\right)-1}{\pi \frac{\exp \left(\pi c_{\theta}\right)-1}{\theta_{N_{\theta}+2-j},},} 1 \leq j \leq \frac{N_{\theta}}{2}+1 \\
2 \pi-\theta_{N_{\theta}}+1 \leq j \leq N_{\theta}+1\end{cases}
\end{aligned}
$$


where $N_{r}, N_{\theta} / 2$ are strictly positive integers and $c_{\theta}$ is a strictly negative real value used to adjust the mesh size in the upper part of the annulus where small convective cells may develop. A time splitting method is adopted to uncouple the velocity and pressure fields [23]. Time evolution is achieved by a first-order Euler scheme, by an implicit treatment of the diffusion terms, except those involving the coupling of velocity components (Eq. (2d)) that are explicitly approximated, as well as all nonlinear terms.

\subsubsection{Steady state and eigenvalue problems}

The steady flow, solution of the non-linear system (4) with boundary conditions (3), is computed by the iterative Newton algorithm. The resulting tangent linear problem is also solved with an iterative technique, namely the Bi-Conjugate Gradient Stabilized method. The use of an iterative process for solving linear systems avoids the explicit construction of tangent matrices. Indeed, only the product of this matrix with a vector is required. That is easily performed by the method proposed by Mamun and Tuckerman [24]. The left-hand side (matrix-vector product) and the right-hand side (vector) of each linear system are evaluated in a similar manner, by using specifically modified time-marching codes which are derived from the governing equations. Details concerning the implementation can be found in a previous paper dealing with the stability of natural convection flows in horizontal annuli [4].

In order to characterize the stability of the basic flow, a search for infinitesimal disturbances having the largest growth rate is carried out. In what follows, we denote the largest growth rate by $\lambda_{r}^{M}=\max _{\lambda_{r} \in s p_{r}}\left(\lambda_{r}\right)$ where $s p_{r}$ denotes the set of the real parts of the eigenvalues for problem (8); the pulsation $\lambda_{i}^{M}$ is the imaginary part of the complex eigenvalue whose real part is $\lambda_{r}^{M}$. Two cases must be considered according to the sign of $\lambda_{r}^{M}$ : the inequality $\lambda_{r}^{M}>0$ guarantees an instability condition for the basic flow, whereas $\lambda_{r}^{M}<0$ ensures stability with respect to small disturbances only (while it tells nothing about finite amplitude perturbations).

For a given set of the dimensionless parameters $\operatorname{Pr}$, Ra and Pe and for a fixed dimensionless wavenumber $k$, computation of the largest growth rate $\lambda_{r}^{M}$ with its associated pulsation $\lambda_{i}^{M}$ is achieved by solving the eigenvalue problem (8) with the free software ARPACK [25] which is based upon an algorithmic variant of the Arnoldi process [26]. This software is designed to compute some eigenvalues of largest real part or largest magnitude and the corresponding eigenvectors of a generic $n$ by $n$ matrix $\mathcal{A}$. In our case, $\mathcal{A}$ is a complex matrix and the dimension of the Krylov subspace used by the Arnoldi method is typically 40. The iterative process then provides between 30 and 40 converged eigenvalues with largest real part. Let a complex variable $x^{(0)}=\left(\delta \boldsymbol{v}_{k, \lambda}, \delta T_{k, \lambda}\right)^{(0)}$ provided by $A R P A C K[25]$, we have to compute the product between the discrete tangent operator $\mathcal{A}$ and $x^{(0)}$. This computation is achieved with a first order time approximation of equations (5), supplied with boundary conditions (7a): the difference between two successive iterations $x^{(1)}-x^{(0)}$ provides a good guess of $\exp (\mathcal{A} \delta t) x^{(0)}-x^{(0)}$ which in turn is a good estimate of $\mathcal{A} x^{(0)} \delta t$ for small $\delta t$ (further details can be found in Petrone et al. [4]). 


\subsubsection{Critical values - Algorithm}

The estimate of the threshold Rayleigh number value $\mathrm{Ra}_{c}$ is achieved by an iterative procedure where the Péclet and Prandtl numbers are assumed to be given. For a wavenumber $k$, we first search $\widehat{\mathrm{Ra}}$ such that $\lambda_{r}^{M}(\widehat{\mathrm{Ra}}, k)=0$. Afterwards, the threshold Rayleigh number value $\mathrm{Ra}_{c}$ is defined by $\operatorname{Ra}_{c}=\min _{k}(\widehat{\operatorname{Ra}}(k))$ or $\operatorname{Ra}_{c}=\max _{k}(\widehat{\operatorname{Ra}}(k))$ depending on whether the basic flow is destabilized or stabilized when increasing Ra. As it is customary, the critical wavenumber $k_{c}$ is defined by $k\left(\mathrm{Ra}_{c}\right)$. In practice, this optimization problem is substituted by the calculation of the local optimum $\left.[d \widehat{R} a / d k]\right|_{k_{c}}=0$. To compute $\mathrm{Ra}_{c}$ and $k_{c}$, the following iterative algorithm is applied.

Let $\tilde{k}_{0}$ and $\widehat{\text { Ra }}$ be some approximations of the critical wavenumber $k_{c}$ and Rayleigh number $\operatorname{Ra}_{c}\left(k_{c}\right)$, and $\epsilon_{\tilde{k}}, \epsilon_{\lambda_{r}^{M}}$ and $\epsilon_{\widehat{\mathrm{Ra}}}$ three small positive real values used as stopping criteria such that $\epsilon_{\tilde{k}}>\epsilon_{\widehat{\mathrm{Ra}}}$. Set $n=0, \widehat{\operatorname{Ra}}_{0}^{i}=\widehat{\mathrm{Ra}}$ for $i=-1,0,1$ and do

1. Solve six steady state problems (4) defined by $\mathrm{Ra}=\widehat{\mathrm{Ra}}_{n}^{i}$ and $\mathrm{Ra}=\widehat{\mathrm{Ra}}_{n}^{i}+\delta \mathrm{Ra}^{i}$ $\left(\delta \mathrm{Ra}^{i} / \widehat{\operatorname{Ra}}_{n}^{i} \ll 1\right)$ with $i=-1,0,1$;

2. Let us consider a set of three dimensionless wavenumbers $k_{n}^{i}=\tilde{k}_{n}+i \delta k$ for $i=$ $-1,0,1\left(\delta k / \tilde{k}_{n} \ll 1\right)$ and compute six eigenvalue problems defined by $\lambda_{r}^{M}\left(\widehat{\operatorname{Ra}}_{n}^{i}, k_{n}^{i}\right)$ and $\lambda_{r}^{M}\left(\widehat{\operatorname{Ra}}_{n}^{i}+\delta \operatorname{Ra}^{i}, k_{n}^{i}\right)$, with $i=-1,0,1$, by solving equation (8);

3. For each wavenumber $k_{n}^{i}, i=-1,0,1$, compute the new approximation $\widehat{\operatorname{Ra}}_{n+1}^{i}$ of the critical Rayleigh number through the relation

$$
\text { For } i=-1,0,1 \text { do }\left\{\begin{array}{l}
\frac{\lambda_{r}^{M}\left(\widehat{\operatorname{Ra}}_{n}^{i}+\delta \operatorname{Ra}^{i}\right)-\lambda_{r}^{M}\left(\widehat{\operatorname{Ra}}_{n}^{i}\right)}{\widehat{\operatorname{Ra}}_{n+1}^{i}=\widehat{\operatorname{Ra}}_{n}^{i}+\Delta \operatorname{Ra}^{i}=-\lambda_{r}^{M}\left(\widehat{\operatorname{Ra}}_{n}^{i}\right)}
\end{array}\right.
$$

4. if $\lambda_{r}^{M}\left(\widehat{\operatorname{Ra}}_{n}^{i}\right)>\epsilon_{\lambda_{r}^{M}}$ or $\Delta \mathrm{Ra}^{i} / \widehat{\operatorname{Ra}}_{n+1}^{i}>\epsilon_{\widehat{\mathrm{Ra}}}$ go to step 1 , otherwise go to step 5;

5. Correct the wavenumber $\tilde{k}$ by solving

$$
\left\{\begin{array}{l}
\frac{\widehat{\mathrm{Ra}}_{n+1}^{1}-2 \widehat{\mathrm{Ra}}_{n+1}^{0}+\widehat{\mathrm{Ra}}_{n+1}^{-1}}{\delta k^{2}} \Delta k=-\frac{\widehat{\mathrm{Ra}}_{n+1}^{1}-\widehat{\mathrm{Ra}}_{n+1}^{-1}}{2 \delta k} \\
\tilde{k}_{n+1}=\tilde{k}_{n}+\Delta k
\end{array}\right.
$$

6. if $\Delta k / \tilde{k}_{n+1}>\epsilon_{\tilde{k}}$ go to step 1, otherwise $\mathrm{Ra}_{c}=\widehat{\operatorname{Ra}}_{n+1}^{0}$ and $k_{c}=\tilde{k}_{n+1}$; stop.

It should be noted that the systems of linear equations (10) and (11) result from applications of the Newton method to solve $\lambda_{r}^{M}(\widehat{\mathrm{Ra}})=0$, for a given $k$-value, and $\left.[d \widehat{\mathrm{Ra}} / d k]\right|_{k_{c}}=0$ where derivatives were approximated by algebraic relations resulting from Taylor expansions. A necessary condition for convergence of the iterative algorithm is that $\delta k \ll \Delta k$ and $\delta \mathrm{Ra}^{i} \ll \Delta \mathrm{Ra}^{i}$, otherwise it may fail. Note also that steady state and eigenvalue computations have to be accurate enough in order to avoid troubles in convergence of the approximated Newton methods (10)-(11). At last, one further information can be added: computations of the six steady flows (step 1) and solution of the six eigenvalue problems (step 2) can be performed simultaneously on different computers or processors. 


\section{Results}

The results were obtained for a fixed radius ratio, $R=1.2$, and for air, $\operatorname{Pr}=0.7$. The mesh is similar to that used in natural convection problems [4], namely 60 regular cells in the radial direction and 240 cells in the azimuthal direction, with a finer mesh at the top of the annular space $\left(c_{\theta}=-0.75\right.$ in Eq. (9)). Preliminary numerical tests indicated that the chosen grid enables to achieve solutions almost insensitive to extra mesh refinements. The critical Rayleigh number was computed as a function of the Péclet number, starting from the natural convection problem.

For the linear stability analysis, we first need the calculation of the steady flows. They consist of the superimposition of two motion fields: the first one corresponds to the main flow which is mainly driven by the axial pressure gradient (see Eq. (4c)), the secondary flow, so-called because it develops in any transverse section of the annular duct, is due to the buoyancy effect induced by the temperature difference imposed between the solid boundaries (see Eqs. (4b), and (4d) and boundary conditions (3)). Thus, except the axial component of the velocity, these secondary flows are identical to those obtained in free convection, namely for $\mathrm{Pe}=0$. The third velocity component, $w_{0}$, is simply the solution of a transport equation wherein the source term is provided by the axial pressure gradient.

As for the natural convection problem [4], the steady flows are shared out on two different branches of solutions. Figure 2 illustrates the bifurcation diagram for the free convection problem: the dimensional radial velocity component of the basic flow at $r=0.5$ and $\theta=\pi$ is rescaled with respect to the thermal diffusion velocity $\left(u_{0}(0.5, \pi) \times \mathrm{Pe}\right)$, and then drawn as a function of Ra. While this velocity scaling aims to provide curves insensitive to the Péclet number, it should be emphasized that the stability property of the solution depends on Pe, as it will be shown in sections 3.1 and 3.2. To easily distinguish the two branches, the Rayleigh number is labeled either by ${ }^{1 \text { st }}$ or by ${ }^{2 n d}$ depending on whether the corresponding flow can be computed or not by means of continuous increasing of the Rayleigh number from zero. Thus, the first branch starts from $\mathrm{Ra}^{1 \mathrm{1st}}=0$ and it is characterized by a secondary flow which firstly consists of two crescent shaped cells (labeled C2, Fig. 3(a)) for Ra ${ }^{1 \text { st }} \lesssim \operatorname{Ra}_{f_{1}}^{1 \text { st }}=2068$, and then of a hexa-cellular motion field, made of two additional couples of convective rolls located above the crescent shaped ones (labeled C6, Fig. 3(b)) for $\operatorname{Ra}^{1 \text { st }} \gtrsim \operatorname{Ra}_{f_{2}}^{1 s t}=2456$. The secondary tetra-cellular flow (labeled $C 4$, Fig. 3(c)) belongs to the second branch of solutions which emerges from $\mathrm{Ra}^{2 \mathrm{nd}} \gtrsim \mathrm{Ra}_{s}^{2 \text { nd }}=1911$ through a saddle-node bifurcation. It may be noted that the fluid flow with two transverse cells, C2, is consistent with what Mojtabi and Caltagirone [22] have presented for larger radius ratios.

For the sake of clarity, the presentation of the stability results for the two branches of solutions is divided into two sections. A third section is then devoted to present the stability diagram for the different established mixed convection flows in the Rayleigh-Péclet plane and to illustrate the characteristics of the marginal perturbations.

\subsection{First branch of basic solutions}

In natural convection, the two-crescent shaped flow pattern (Fig. 3(a)) and the hexacellular one (Fig. 3(b)) are found stable for two-dimensional perturbations [4] when Ra is 


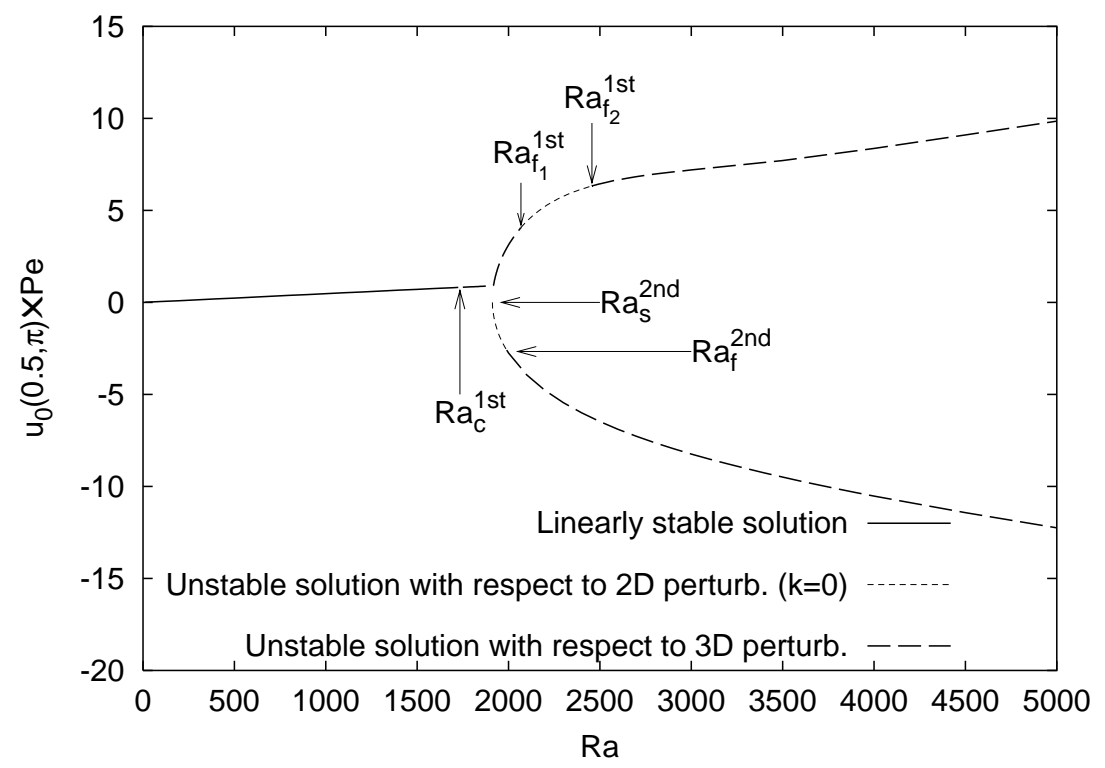

Figure 2: Bifurcation diagram for natural convection. Dimensional radial velocity component rescaled with respect to the thermal diffusion velocity, $u_{0}(0.5, \pi) \times \mathrm{Pe}$, as a function of Ra. $\mathrm{Ra}_{c}^{1 \text { st }}=1734, \mathrm{Ra}_{f_{1}}^{\text {sst }}=2068$, $\mathrm{Ra}_{f_{2}}^{1 \text { st }}=2456, \mathrm{Ra}_{s}^{2 \text { nd }}=1911, \mathrm{Ra}_{f}^{2 \text { nd }}=2000$.

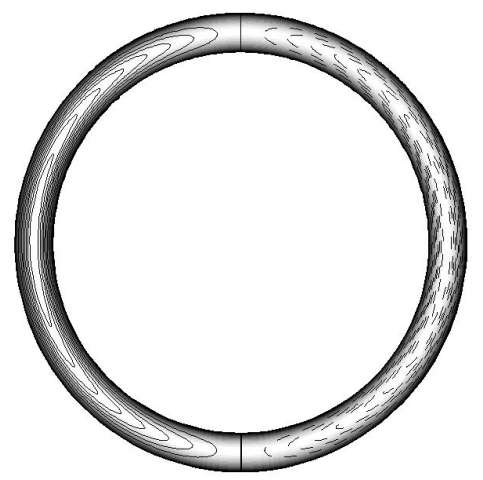

(a) Two crescent shaped flow labeled $C 2\left(\mathrm{Ra}^{\text {1st }}=1700\right)$.

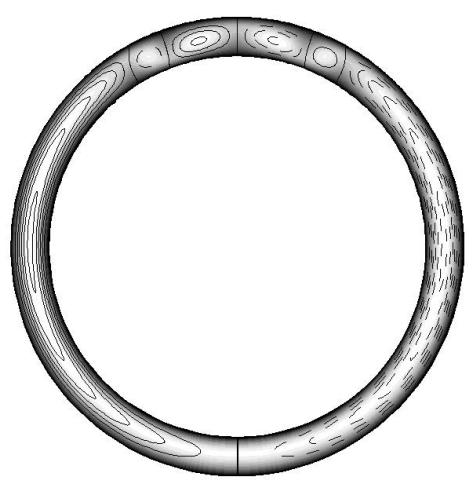

(b) Hexa-cellular flow $C 6$, $\left(\mathrm{Ra}^{1 \mathrm{st}}=5000\right)$.

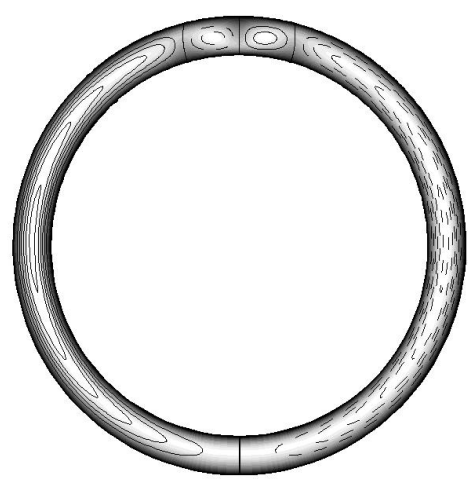

(c) Tetra-cellular flow labeled $C 4,\left(\mathrm{Ra}^{2 \mathrm{nd}}=5000\right)$.

Figure 3: Representative examples of steady and fully developed flows which occur for (a) $\mathrm{Ra}^{1 \text { st }} \lesssim \mathrm{Ra}_{f_{1}}^{1 \text { st }}=$ 2068, (b) $\mathrm{Ra}^{1 \mathrm{1st}} \gtrsim \mathrm{Ra}_{f_{2}}^{1 \text { st }}=2456$ and (c) $\mathrm{Ra}^{2 \text { nd }} \gtrsim \mathrm{Ra}_{s}^{2 \text { nd }}=1911$. Streamlines and axial velocity field.

respectively lower and higher than two thresholds, identifying a supercritical $\left(\operatorname{Ra}_{f_{1}}^{1 \text { st }}=2068\right)$ 
and a sub-critical $\left(\mathrm{Ra}_{f_{2}}^{1 \text { st }}=2456\right)$ pitchfork bifurcation; in-between, the steady flows exhibit intermediate unstable patterns. Because the stability problem (5), with $k=0$, is identical to the one obtained for free convection problem, the range $\operatorname{Ra}_{f_{1}}^{1 \text { st }}<\mathrm{Ra}^{1 \text { st }}<\mathrm{Ra}_{f_{2}}^{1 \text { st }}$ is then also unstable for any established axial flow. Now, by considering any three-dimensional perturbation, the natural convection is linearly stable only for $\mathrm{Ra}^{1 \text { st }}<\mathrm{Ra}_{c}^{1 \text { st }}(\mathrm{Pe}=0)=1734$, with a non-oscillatory marginal mode characterized by a wavenumber $k_{c}(\mathrm{Pe}=0)=3.04$ $[5,6]$.

Figure 4 shows different schematic continuation curves obtained by increasing the Péclet

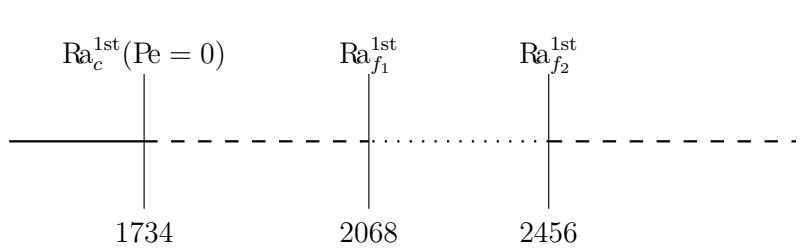

(a) $\mathrm{Pe}=0$

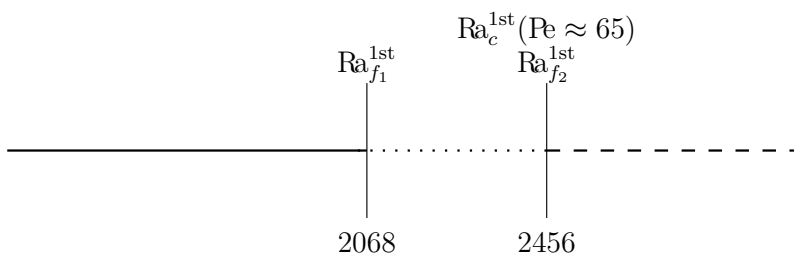

(c) $\mathrm{Pe} \approx 65$

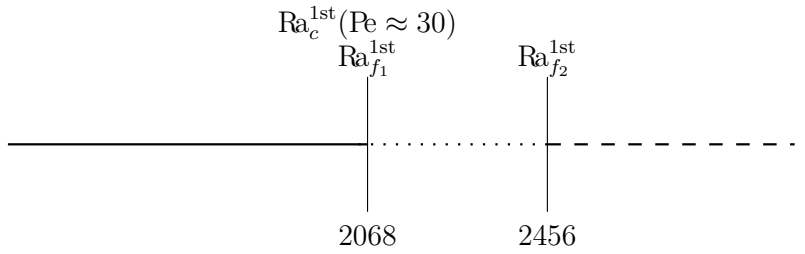

(b) $\mathrm{Pe} \approx 30$

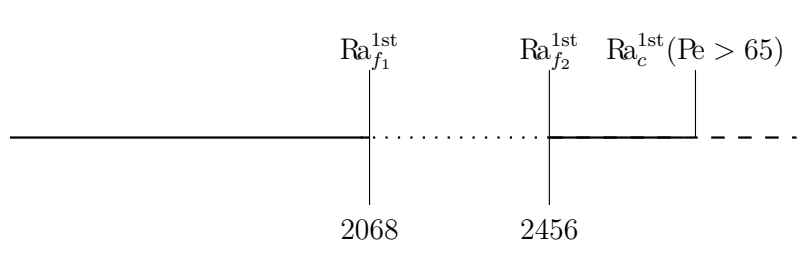

(d) $\mathrm{Pe}>65$

Figure 4: Schematic continuation curves for the first branch of steady solutions as a function of Ra, for (a) $\mathrm{Pe}=0$, (b) $\mathrm{Pe} \approx 30$, (c) $\mathrm{Pe} \approx 65$ and (d) $\mathrm{Pe}>65$. Continuous lines stand for linearly stable solutions, dashed lines indicate unstable solutions with respect to three-dimensional perturbations and dotted lines specify instabilities also obtained for two-dimensional perturbations.

number: continuous lines stand for linearly stable solutions, dotted lines indicate unstable solutions with respect to uniform axial disturbances $(k=0)$, and dashed lines illustrate instabilities to three-dimensional perturbations. By increasing the Péclet number from $\mathrm{Pe}=$ 0 (Fig. 4(a) and the bifurcation diagram in Fig. 2) to about $\mathrm{Pe}=30$ (Fig. 4(b)), Ra ${ }_{c}^{1 \text { st }}$ moves from 1734 to $R a_{f_{1}}^{1 \text { st }}=2068$. Then, the critical Rayleigh number value remains unchanged as long as $\mathrm{Pe} \lesssim 65$ (Fig. 4(c)). For $30 \lesssim \mathrm{Pe} \lesssim 65$, the transition threshold is given by the unstable perturbations which are uniform in the axial direction (see the dotted lines on the continuation curve in Fig. 4). Once Pe $>65$, a new stable region appears for $\operatorname{Ra}_{f_{2}}^{1 \mathrm{st}}<\mathrm{Ra}^{1 \mathrm{st}}<\operatorname{Ra}_{c}^{1 \mathrm{st}}=\min _{k}\left(\widehat{\operatorname{Ra}}^{1 \mathrm{st}}(k, \mathrm{Pe}>65)\right)($ Fig. $4(\mathrm{~d}))$.

For a better understanding of the relation between the critical Rayleigh and Péclet number values, we also present the qualitative evolution of $\widehat{\mathrm{Ra}}^{1 \mathrm{st}}(k)$, which corresponds to a zero growth rate of disturbances, as a function of the wavenumber $k$, for specific Pe-values (Fig. 5). Stable (respectively unstable) regions are labeled with the upper case $S$ (respectively $U$ ). The hatched region denotes unstable flows, at least for two dimensional perturbations. In the 


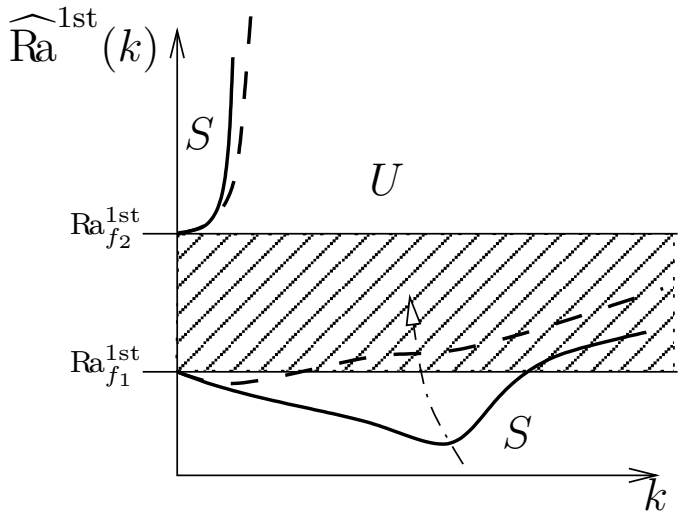

(a) $0 \leq \mathrm{Pe} \lesssim 30$

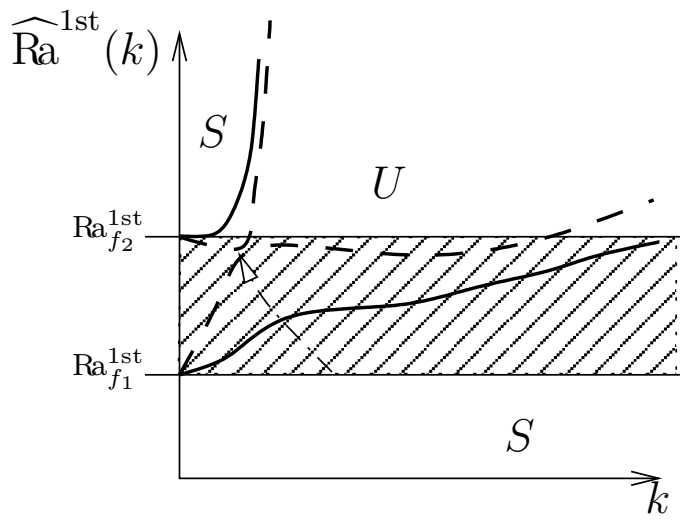

(b) $60<\mathrm{Pe}<65$

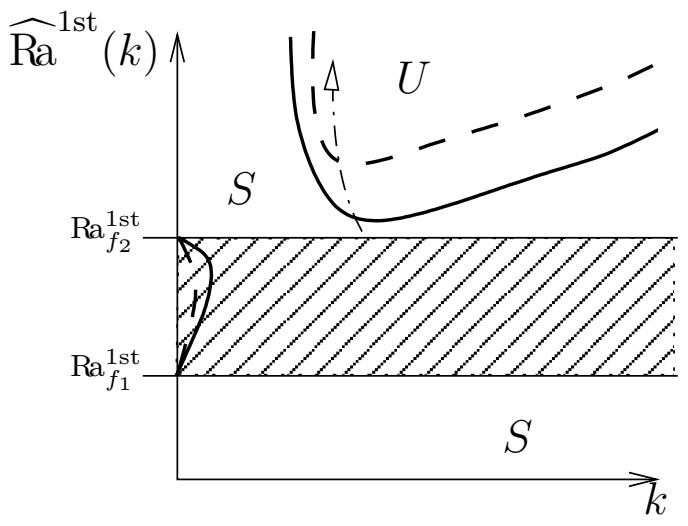

(c) $\mathrm{Pe}>65$

Figure 5: Schematic modifications of $\widehat{\mathrm{Ra}}^{1 \mathrm{st}}(k)$ for different Pe range of variation. The hatched region indicates the unstable steady states against two-dimensional disturbances $(k=0)$. By increasing the Pe-value, the continuous curves $\widehat{\operatorname{Ra}}^{1 \text { st }}(k)$ move according to the directions showed by the dashed-dotted arrows, in order to give rise to separated unstable regions. Upper case letters $U$ and $S$ emphasize the unstable or stable condition of the established steady flow.

natural convection problem $(\mathrm{Pe}=0)$, two stability curves represented by continuous lines in Fig. 5(a) begin from points $\left(0, \mathrm{Ra}_{f_{1}}^{1 \text { st }}\right)$ and $\left(0, \mathrm{Ra}_{f_{2}}^{1 \text { st }}\right)$. The stable flow regions are located below the lower curve and in the upper left portion of the plane bounded by the upper marginal stability curve. By increasing the Péclet number in the range $60<\mathrm{Pe}<65$, the following scenario occurs: the stability curves get more and more closer, they join together before to separate again in the form of two new stability curves. Beyond $\mathrm{Pe}=65$, one of the two curves links the two points $\left(0, \mathrm{Ra}_{f_{1}}^{1 \mathrm{st}}\right)$ and $\left(0, \mathrm{Ra}_{f_{2}}^{1 \text { st }}\right)$, while the second curve is, from now on, released from any fixed point in the $\left(\widehat{\mathrm{Ra}}^{1 \mathrm{st}}, k\right)$-plane. As a result, this second curve is free to move upwards and a new stable region defined by $\operatorname{Ra}_{f_{2}}^{1 \mathrm{st}}<\operatorname{Ra}^{1 \mathrm{st}}<\operatorname{Ra}_{c}^{1 \mathrm{st}}=\min _{k}\left(\widehat{\operatorname{Ra}}^{1 \mathrm{st}}(k, \mathrm{Pe}>65)\right)$ emerges. 


\subsection{Second branch of basic solutions}

As mentioned at the beginning of this section, the tetra-cellular flows (Fig. 3(c)) are located on a second branch of solutions which emerges from a saddle-node bifurcation at $\mathrm{Ra}^{2 \text { nd }} \gtrsim \mathrm{Ra}_{s}^{2 \text { nd }}=1911$ (Fig. 6 or bifurcation diagram in Fig. 2). By using the same labels

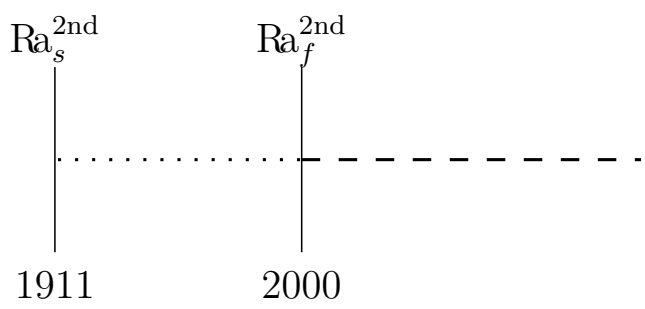

(a) $\mathrm{Pe} \lesssim 25$

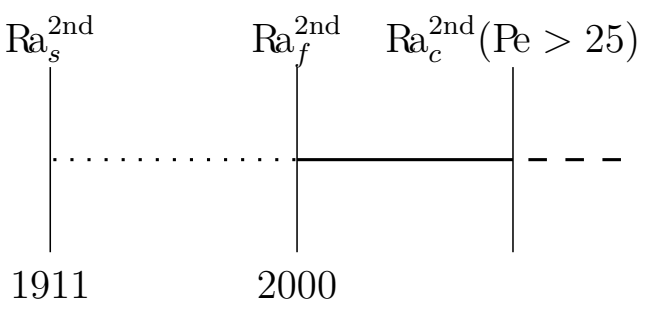

(b) $\mathrm{Pe}>25$

Figure 6: Schematic continuation curves for the lower branch of steady solutions as a function of Ra, for (a) $\mathrm{Pe} \lesssim 25$ and (b) Pe $>25$. Continuous lines stand for linearly stable solutions, dashed lines indicate unstable solutions with respect to three-dimensional perturbations and dotted lines specify instabilities also obtained for two-dimensional perturbations.

for lines drawn in Fig. 4, dotted lines indicate unstable established flows with respect to two-dimensional perturbations $(k=0)$ and dashed lines symbolize instability of basic flows against three-dimensional disturbances. In agreement with the published results for natural convection [5, 6], solutions are always unstable as long as $\mathrm{Pe} \lesssim 25$. For $\mathrm{Pe}>25$, a small stability region defined by $\operatorname{Ra}_{f}^{2 \text { nd }} \simeq 2000<\operatorname{Ra}^{2 \text { nd }}<\operatorname{Ra}_{c}^{2 \text { nd }}=\min _{k}\left(\widehat{\operatorname{Ra}}^{2 \text { nd }}(k, \mathrm{Pe}>25)\right)$ appears and extends with increasing Péclet. The lower boundary $\mathrm{Ra}_{f}^{2 \text { nd }}$ for the stability region of the tetra-cellular flows is defined by a pitchfork bifurcation with a wavenumber equal to zero [4].

The large modifications in the continuation curve become clear by analyzing the change in the shape of the stability curves $\widehat{\mathrm{Ra}}^{\text {nd }}(k)$ when the Pe value is increased (Fig. 7). For $0 \leq \mathrm{Pe} \lesssim 10$ (Fig. 7(a)), two continuous lines can be distinguished. The first one is anchored at the pitchfork two-dimensional bifurcation at $\left(k=0, \mathrm{Ra}_{f}^{2 n d}\right)$. The second one is free to move, and it crosses the saddle-node bifurcation at $\mathrm{Ra}_{s}^{2 \text { nd }}$. Increasing Pe leads to a shift of the stability curve: that is emphasized by the dashed lines drawn in the same figure 7(a). As the arrows indicate, the curve lying on the right side undergoes a rotation toward larger Ra-values and lower wavenumbers. In Fig. 7(b) it is shown how that curve continues to move upward and to the left, to finally go closer and closer to the stability curve extending from the fixed point $\left(k=0, \mathrm{Ra}_{f}^{2 \text { nd }}\right)$. For $\mathrm{Pe} \approx 25$, the two curves combine together before getting reorganized so that the stability curve connected to the point $\left(0, \mathrm{Ra}_{f}^{2 \text { nd }}\right)$ is, from now on, a decreasing function of $\mathrm{Ra}$ (Fig. 7(c)). On the other hand, this reorganization leads to the appearance of a marginal stability curve which can move upward when Pe increases: this allows a stable region for the basic flow to arise. 


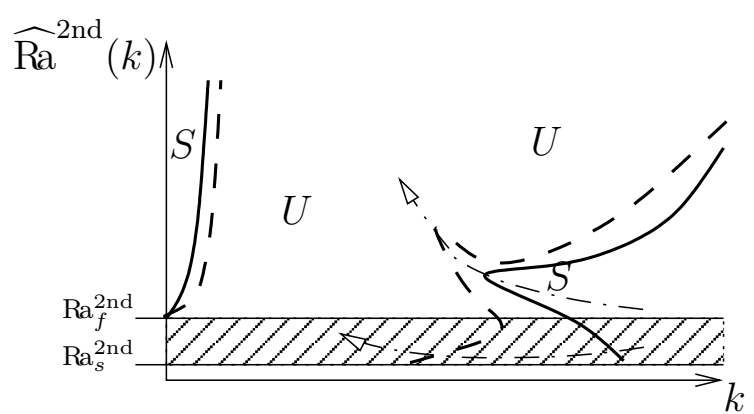

(a) $0 \leq \mathrm{Pe} \lesssim 10$

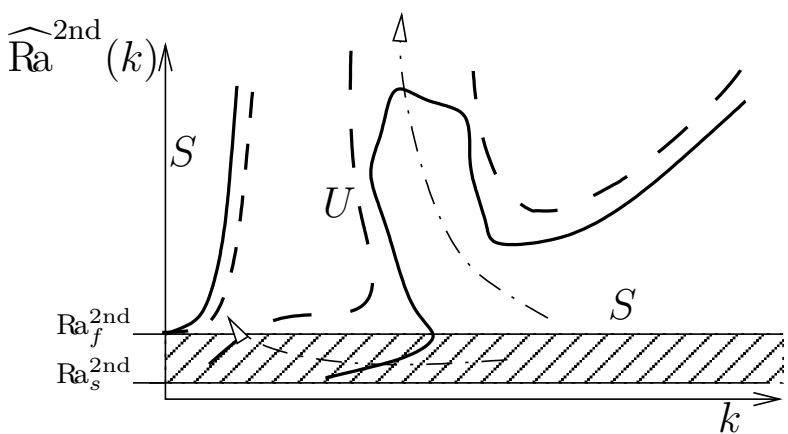

(b) $15 \lesssim \mathrm{Pe} \lesssim 25$

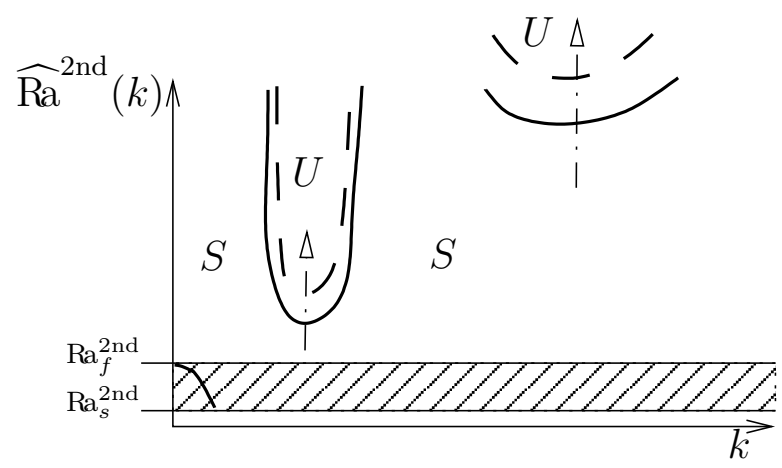

(c) $\mathrm{Pe}>25$

Figure 7: Schematic variation of $\widehat{\mathrm{Ra}}^{2 \text { nd }}(k)$ as a function of Pe. See the legend of Fig. 5 for extra details.

\subsection{Global stability diagram}

Fig. 8 presents the marginal stability curves in the plane of the Rayleigh number and Péclet number, for mixed convection flows which are characterized by three different secondary patterns. The two- and tetra-cellular secondary flows are stable simultaneously in a narrow gap of the Rayleigh number, $2000<\mathrm{Ra}<\mathrm{Ra}_{f_{1}}^{1 \text { st }}=2068$, with Pe $\gtrsim 25$ (region doubly hatched). On the other hand, the co-existence of the tetra- and hexa-cellular secondary flows occurs in a wide range of the parameters, the region filled in gray, and defined by $\mathrm{Pe}>65$ and $\mathrm{Ra}_{f_{2}}^{1 \text { st }}=2456<\mathrm{Ra}<\operatorname{Ra}_{c}^{1 \text { st }}(\mathrm{Pe}>65)$. $\operatorname{Ra}_{c}^{1 \text { st }}(\mathrm{Pe})$ may be approximated by the following quadratic law, accurate within $\pm 0.5 \%$

$$
\mathrm{Ra}_{c}^{1 \mathrm{st}}(\mathrm{Pe})=0.02095 \mathrm{Pe}^{2}+4.951 \mathrm{Pe}+2033, \quad \mathrm{Pe} \geq 65
$$

At last, beyond the hatched regions, the mixed convective flows are linearly unstable. For Pe $\lesssim 25$ (see the enlargement in Fig. 8), the marginal Rayleigh curve, which bounds the stability region of fluid flows made of two-crescent shaped cells, is more complex so that no relevant analytical approximation is given. In contrast, for Pe greater than about 25, the marginal stability curve for mixed convection flows is reasonably approximated by the analytical function

$$
\operatorname{Ra}_{c}^{2 n d}(\mathrm{Pe})=34.2 \mathrm{Pe}+1200, \quad \mathrm{Pe} \geq 25
$$




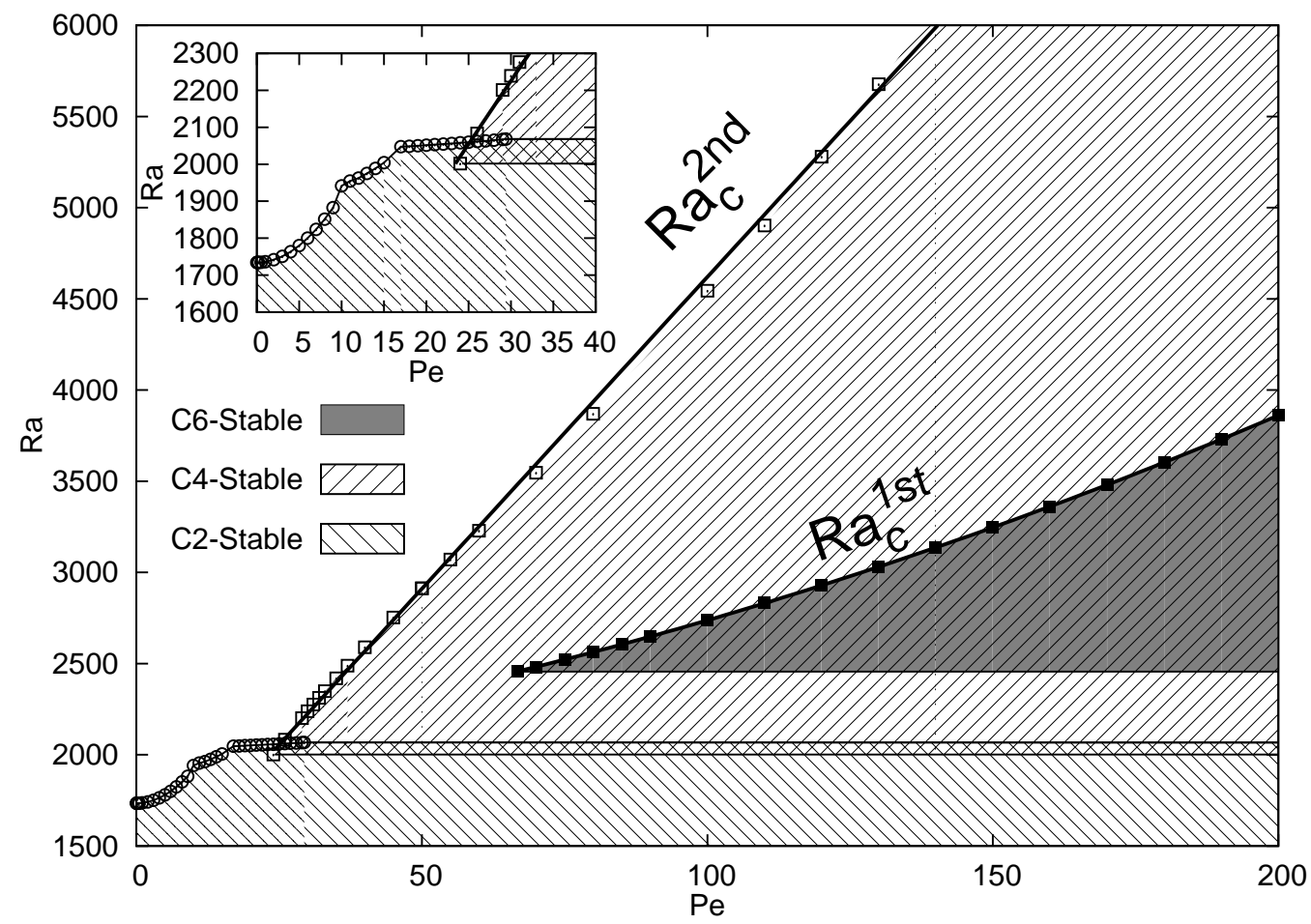

Figure 8: Stability diagram in the plane (Pe, Ra) for mixed convection solutions when secondary flows are made up either of two-cells $(C 2)$, of four cells $(C 4)$ or of six cells $(C 6)$. The labels C2-Stable, C4-Stable or C6-Stable indicate the stability regions of the corresponding secondary flows. Symbols lying on curves are result of numerical simulations. The marginal stability curves $\operatorname{Ra}_{c}^{1 \text { st }}(\mathrm{Pe})(\mathrm{Eq} .12)$ and $\mathrm{Ra}_{c}^{2 n d}(\mathrm{Pe})(\mathrm{Eq}$. 13) are also drawn with thick lines.

accurate within $\pm 2 \%$. By focusing on the marginal curve associated with the $C 2$ pattern (see the enlargement in Fig. 8), we can clearly distinguish two changes in the slope of the curve $\mathrm{Ra}_{c}^{1 \text { st }}(\mathrm{Pe})$ for $\mathrm{Pe}<30$. The first change occurs for $9<\mathrm{Pe}<10$ and $1882<\mathrm{Ra}^{1 \text { st }}<1941$, a Rayleigh interval in which the so-called virtual-transcritical bifurcation [4, 27] (or imperfect pitchfork bifurcation [28]) was pointed out in natural convection, around $\mathrm{Ra}^{1 \mathrm{st}}=1920$. According to the works of Petrone et al. [4], this pseudo-bifurcation indicates the beginning of a large modification of the secondary flow as a function of the Rayleigh number. Later on, these changes will give rise to the appearance of four new recirculation cells at $\mathrm{Ra}^{1 \mathrm{st}} \simeq 2270$ and, therefore to the birth of the $C 6$-secondary flow. Such a $C 6$-secondary flow becomes stable for mixed convection under certain conditions (see Fig. 8). The sudden evolution in the slope of the critical Rayleigh number with respect to Pe is also combined with more or less pronounced discontinuities in the representation of the wavenumber as a function of Pe for the C2-secondary flow (see the circles in Fig. 9 drawn using the left and bottom axes). 


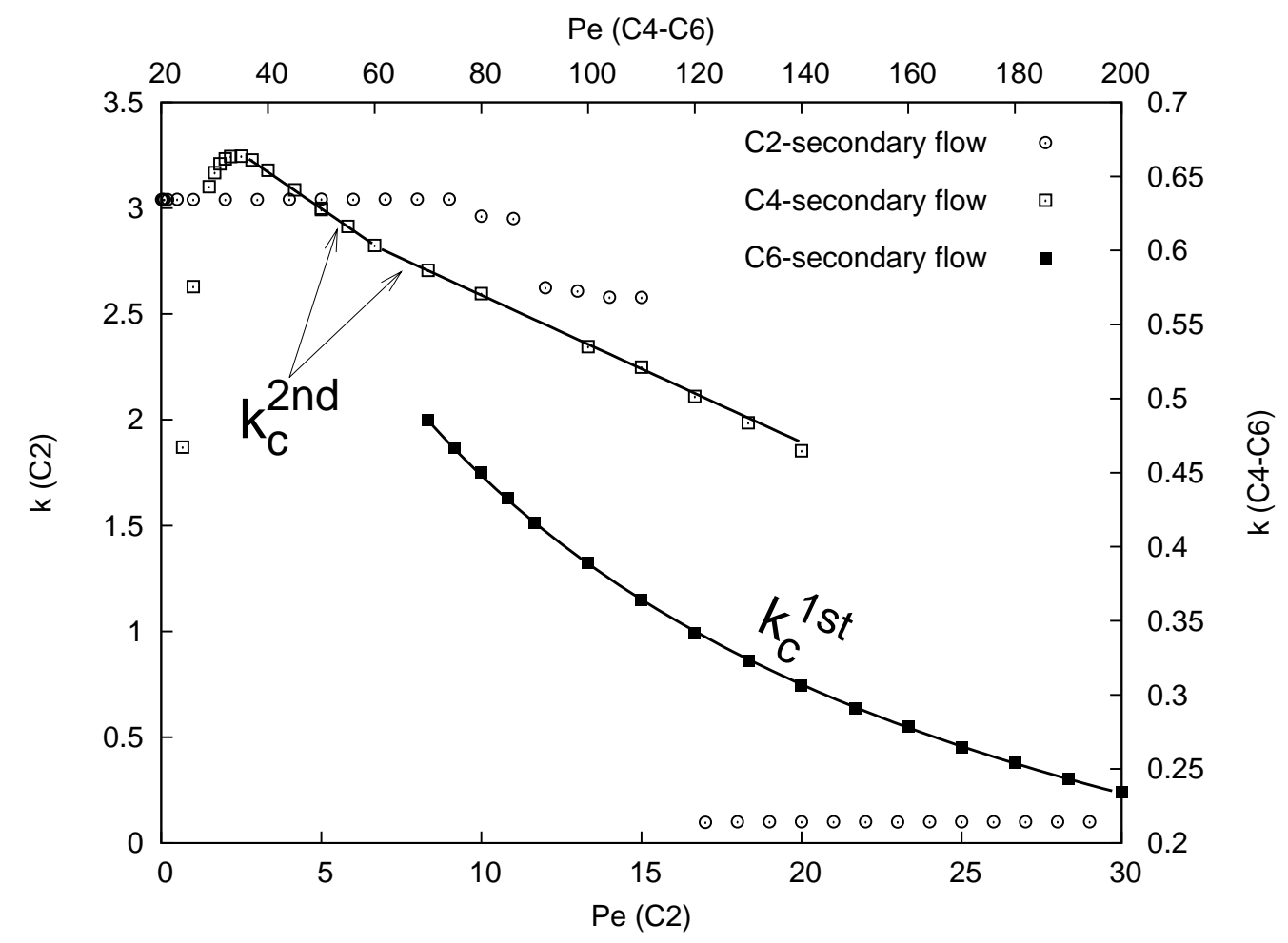

Figure 9: Evolution of the critical wavenumber $k_{c}$ as a function of Pe for the C2-secondary flow (left and bottom axes, with circles) and $C 4-C 6$-secondary flow (right and top axes, with empty and full squares). The analytical approximations $k_{c}^{\text {1st }}(\mathrm{Pe})(\mathrm{Eq} .14)$ and $k_{c}^{2 \text { nd }}(\mathrm{Pe})($ Eq. 15) are drawn with lines.

These jumps are due to the emergence of new global minima in the curve $\widehat{\mathrm{Ra}}^{1 \mathrm{st}}(k)$ (Fig. $5(\mathrm{a})$, dashed curve). Let us recall that our critical values are evaluated by using the algorithm presented in Sec. 2.2.3, a method providing the calculation of maximum or minimum values in a local sense, not in global one as it should be. The direct consequence of the adopted numerical approach is that solutions depend on the initialization of our iterative algorithm when the solution is not unique and therefore it may converge toward a wrong solution. Notice however that for a fixed Péclet value, the critical Rayleigh number is generally less marred by mistakes than the critical wavenumber can be, especially when $\widehat{\mathrm{Ra}}$ varies weakly as a function of the wavenumber. The analytical approximation of the wavenumber as a function of the critical Péclet number can be written as follows:

- on the first branch of solutions and for the $C 6$-secondary flows

$$
k_{c}^{1 \mathrm{st}}(\mathrm{Pe})=\frac{1}{0.01709 \mathrm{Pe}+0.8645}, \quad \mathrm{Pe} \geq 65
$$


- on the second branch of solutions and for the $C 4$-secondary flows

$$
k_{c}^{2 \text { nd }}(\mathrm{Pe})= \begin{cases}-2.468 \cdot 10^{-3} \mathrm{Pe}+0.7517 & \text { if } 35 \leq \mathrm{Pe} \leq 60 \\ -1.657 \cdot 10^{-3} \mathrm{Pe}+0.7024 & \text { if } 60 \leq \mathrm{Pe} \leq 140\end{cases}
$$

with an accuracy within about 5\%o. Note also that we find again a critical wavenumber slightly above 3 for the natural convection problem $(\mathrm{Pe}=0)$, a value consistent with the literature data $[5,6]$.

Once the Péclet number exceeds zero, the dominant perturbation becomes oscillatory

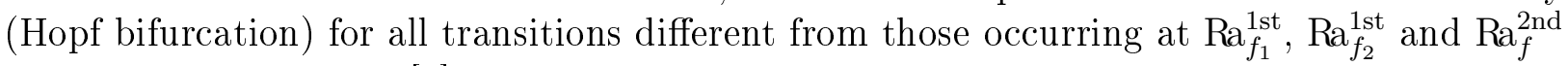
for which Petrone et al. [4] proved that they correspond to pitchfork bifurcations. The phase velocity at the transition, $\lambda_{i}^{M} / k_{c}$, is almost constant, equal to $1.125 \pm 3 \%$. Thus, the linear disturbance propagates approximately $10 \%$ faster that the mean velocity $\bar{w}$ of the main axial flow.

\section{Conclusion}

The linear stability of the fully developed mixed convection flows of air has been numerically investigated for a narrow horizontal cylindrical duct of radius ratio $R=1.2$ in the range $0<\mathrm{Pe}<200$ and $\mathrm{Ra}<6000$. The steady solutions were computed by a Newton algorithm. Both Laplace and Fourier transforms were used to express the time evolution of infinitesimal perturbations and the resulting eigenvalue problem was solved by using the free software ARPACK [25]. The critical Rayleigh and Péclet numbers as well as the wavenumber and frequency were iteratively evaluated by means of approached Newton methods which necessitate successive computations of the basic flow and eigenvalue problem. Our algorithm has been successfully validated on the natural convection problem for which the critical Rayleigh and wavenumber are well established in the literature. For mixed convection problems, in addition to the usual secondary flow consisting of two crescent-shaped cells, we proved that two other flow patterns can also be stable by increasing the Péclet number. These new solutions are characterized by secondary thermal and dynamical fields identical to those encountered in natural convective configurations for a two-dimensional annular space, provided that the parameters are identical. As a result, the instability regions highlighted for 2D natural convection flows are preserved for mixed convection conditions. A stability diagram in the (Pe, Ra)-plane was drawn to define the stability regions of the secondary flows which consist of the two crescent-shaped cells, with zero, two or four extra recirculation cells located at the top of the horizontal annular duct. Analytical expressions for the variations of the critical Rayleigh number and wavenumber were established as a function of the Péclet number. Two regions in the parameters space were pointed out in which two kinds of secondary flows are stable. This result indicates a multiplicity of solutions and thus a probable dependency of the fully developed flow to the initial conditions. 


\section{Acknowledgement}

This work was partially performed using HPC resources from GENCI-[CCRT/CINES/IDRIS] (Grants 2009-021265, 2010-021265).

\section{References}

[1] W. Beckmann, Die Wärmeübertragung in zylindrischen Gasschichten bei natürlicher Konvektion, Forsch. Ingenieurwes. 2 (1931) 165-178.

[2] U. Grigull, W. Hauf, Natural convection in horizontal cylindrical annuli, in: Proc. of the third Int. Heat Transfer Conference, Paper No 60, volume 2, 1966, pp. 182-195.

[3] R. Powe, C. Carley, S. Carruth, A numerical solution for natural convection in cylindrical annuli, J. Heat Transfer 93 (1971) 210-219.

[4] G. Petrone, E. Chénier, G. Lauriat, Stability of free convection in air-filled horizontal annuli: Influence of the radius ratio, Int. J. Heat Mass Transfer 47 (2004) 3889-3907.

[5] J. Choi, M.-U. Kim, Three-dimensional linear stability of natural convective flow between concentric horizontal cylinders, Int. J. Heat Mass Transfer 36 (1993) 4173-4180.

[6] G. Petrone, E. Chénier, G. Lauriat, Stability analysis of natural convective flows in horizontal annuli: effects of axial and radial aspect ratios, Phys. Fluids 18 (2006) 104107-1/14.

[7] G. Petrone, E. Chénier, G. Lauriat, Three-dimensional study of multiple transitions for natural convection in horizontal annuli, Int. J. Heat Mass Transfer 49 (2006) 1231-1241.

[8] T. Adachi, S. Imai, Three-dimensional linear stability of natural convection in horizontal concentric annuli, Int. J. Heat Mass Transfer 50 (2007) 1388-1396.

[9] D. Angeli, G. S. Barozzi, M. W. Collins, O. M. Kamiyo, A critical review of buoyancy-induced flow transitions in horizontal annuli, Int. J. Therm. Sci. 49 (2010) 2231-2241.

[10] W. M. Rohsenow, J. P. Hartnett, Y. I. Cho, Handbook of Heat Transfer, Mc Graw-Hill Handbooks, 3rd edition, 1998.

[11] L. Graetz, Über die Wärmeleitungsfähigkeit von Flüssigkeiten, Ann. Phys. Chem. 18 (1883) 79-94.

[12] W. Nusselt, Die Abhängigkeit der Wärmeübergangszahl von der Rohrlänge, VDI-Z. 54 (1910) 11541158.

[13] M. A. Lévêque, Les lois de la transmission de chaleur par convection, Annales des Mines 13 (1928) 201-299, 305-362, 381-415.

[14] R. E. Lundberg, P. A. McCuen, W. C. Reynolds, Heat transfer in annular passages. Hydrodynamically developed laminar flow with arbitrarily prescribed wall temperatures or heat fluxes, Int. J. Heat Mass Transfer 6 (1963) $495-529$.

[15] R. Shah, A. London, Laminar flow forced convection in ducts, New York: Academic Press, 1978.

[16] S. Kakaç, O. Yücel, Laminar flow heat transfer in an annulus with simultaneous development of velocity and temperature fields, in: Technical and Scientific Concil of Turkey, TURBITAK, ISITEK, Ankara, Turkey, volume 19, 1974.

[17] C.-J. Hsu, Theoretical solutions for low-Péclet-number thermal-entry-region heat transfer in laminar flow through concentric annuli, Int. J. Heat Mass Transfer 13 (1970) 1907 - 1924.

[18] B. Weigand, M. Wolf, H. Beer, Heat transfer in laminar and turbulent flows in the thermal entrance region of concetric annuli: Axial heat conduction effects in fluid, Heat Mass Transfer. 33 (1997) 67-80.

[19] A. Nieckle, S. V. Patankar, Mixed convection in concentric annulus with horizontal axis, J. Heat Transfer 107 (1985) 902-909.

[20] C. Nonino, S. Del Giudice, Finite element analysis of laminar mixed convection in the entrance region of horizontal annular ducts, Num. Heat Transfer, part A 29 (1996) 313-330.

[21] H. Mohammed, A. Campo, R. Saidur, Experimental study of forced and free convective heat transfer in the thermal entry region of horizontal concentric annuli, Int. Commun. Heat Mass Transfer 37 (2010) $739-747$. 
[22] A. Mojtabi, J.-P. Caltagirone, Analyse du transfert de chaleur en convection mixte laminaire entre deux cylindres coaxiaux horizontaux, Int. J. Heat Mass Transfer 23 (1980) 1369-1375.

[23] G. Karniadakis, M. Israeli, S. Orsag, High order splitting method for incompressible Navier-Stokes equations, J. Comput. Phys. 97 (1991) 414-443.

[24] C. Mamun, L. Tuckerman, Asymmetry and Hopf bifurcation in spherical Couette flow, Phys. Fluids 7 (1995) 80-91.

[25] R. Lehoucq, D. Sorensen, C. Yang, ARPACK users' guide: solution of large-scale eigenvalue problems with implicitly restarted Arnoldi methods, SIAM, 1998. ARPACK: http://www.caam.rice.edu/software/ARPACK.

[26] W. Arnoldi, The principle of minimized iterations in the solution of the matrix eigenvalue problem, Q. Appl. Math. 9 (1951) 17-29.

[27] J. Mizushima, S. Hayashi, T. Adachi, Transitions of natural convection in a horizontal annulus, Int. J. Heat Mass Transfer 44 (2001) 1249-1257.

[28] P. Cadiou, G. Desrayaud, G. Lauriat, Natural convection in a narrow horizontal annulus: the effects of thermal and hydrodynamic instabilities, J. Heat Transfer 120 (1998) 1019-1026. 